\title{
3D-printed biodegradable gyroid scaffolds for tissue engineering applications
}

Loïc Germain ${ }^{a, b}$, Carlos A. Fuentes ${ }^{c}$, Aart W. van Vuure ${ }^{c}$, Anne des Rieux ${ }^{a, b,{ }^{*}}$, Christine Dupont-Gillain ${ }^{b,{ }^{*}}$

${ }^{a}$ Université catholique de Louvain, Louvain Drug Research Institute, Advanced Drug Delivery and Biomaterials, Avenue E. Mounier 73, 1200 Bruxelles, Belgium

${ }^{b}$ Université catholique de Louvain, Institute of Condensed Matter and Nanosciences, Place L. Pasteur 1 (L4.01.10), 1348 Louvain-la-Neuve, Belgium

${ }^{c}$ KU Leuven, Department of Materials Engineering, 3001 Leuven, Belgium

*Corresponding authors

\begin{abstract}
Fused deposition modeling (FDM), a low cost and easy-to-use additive manufacturing technique, was pushed to its typical resolution limit to produce poly(lactic acid) (PLA) gyroid scaffolds. A gyroid morphology was selected as scaffold structure due to its spring shape architecture, high porosity, leading to good nutrient and waste diffusion, and favorable mechanical properties, such as isotropic resistance to pressure. Printing parameters were optimized and the need of a support material to improve printing quality was evidenced. The gyroid structure was compared with the more common strut-based structure. Scaffold porosity was measured by micro-CT, and mechanical properties were determined by compressive mechanical tests. The effect of mesh geometry, printing resolution, and PLA crystallinity on resistance against compression was evaluated. Moreover, the impact of PLA scaffold geometry and crystallinity on its degradation was studied in vitro. Porosity of the gyroid structure was $71 \%$, close to the $74 \%$ expected from the model used for printing. The compression tests showed that the gyroid scaffold has an isotropic behavior, in contrast with the typical strut-based scaffold, which exhibits an orientation-dependent deformation. Upon aging in physiological conditions, gyroid scaffolds retained their integrity during 64 weeks, while control scaffolds lost struts one after the other starting from week 33 , in a way that depended on crystallinity and printing resolution. For both geometries, the remaining mass started to decrease at week 52. Based on these
\end{abstract}


results, the gyroid design is proposed as a suitable mesh architecture for tissue engineering scaffolds that can be elaborated using FDM techniques, to produce low cost and personalized implants.

\section{Keywords}

Fused Deposition Modelling; poly(lactic acid); gyroid; scaffold 


\section{Introduction}

Tissue engineering is an interdisciplinary field linking engineering and biology in order to recover, maintain or improve the function of a tissue or organ [1]. It focuses on three main actors: cells, biomaterials and bioactive factors, that are combined to mimic the physical and chemical structure of the body [2]. These actors are implanted or injected to stimulate the regeneration of a damaged tissue. Cells, usually stem cells, proliferate, differentiate and form the new tissue or, at least, improve the recovery of the lesion, notably owing to their unique immunomodulatory properties [3-5]. The biomaterial will generally be processed as a scaffold, to which bioactive compounds can be added, with a view to carry cells and guide their behavior $[6,7]$.

The physical properties of the scaffold are of upmost importance. The mechanical properties have to fit with the ones of the implantation site. For instance, a too soft scaffold could lose its primary shape due to compression by native tissues [8], as is the case for musculoskeletal implantations. For applications such as nasal implants, flexibility is however needed to avoid complications such as infection or extrusion [9]. The scaffold design can also impact cell attachment, proliferation, migration and differentiation $[10,11]$. One important parameter influencing these cell behaviors is porosity. Indeed, pore size and interconnectivity determine communication among cells as well as nutrient, gas and waste diffusion $[6,11]$. Too small pores and a lack of interconnectivity could lead to cell necrosis [8].

Scaffolds meeting these requirements can be produced with a range of different techniques such as salt leaching, gas foaming, fiber bonding, solvent casting, melt molding, phase separation, freeze drying, photolithography and electrospinning [6, 8]. Besides, Solid Freeform Fabrication (SFF) methods are easier to implement to reach designs with controlled dimensions, complex shapes and high porosity. This is ensured by the layer-by-layer construction process which relies on computer-aided design/computer-aided manufacturing (CAD/CAM) systems. SFF methods do not require the use of a mold, and models can be directly generated from computerized tomography, laser scanning or 
magnetic resonance imaging data, allowing the production of personalized implants [12]. Moreover, complex shapes that could not be obtained with molding can be produced based on layer-by-layer construction. Unfortunately, most SFF techniques are expensive. Fused Deposition Modeling (FDM) is however a low cost and easy-to-use SFF method [10]. This nozzle-based technique is based on the layer-by-layer deposition of thermoplastic polymers in a semi-liquid state along an extrusion path. It can be applied to a large variety of materials, and allows the production of patient-personalized scaffolds with a precise control of the geometry, with a typical resolution of $0.4 \times 0.4 \times 0.1 \mathrm{~mm}^{3}[10$, $13,14]$

Polymers, owing to their versatile mechanical properties and their ease of processing, have been extensively used as biomaterials in the tissue engineering field [10]. They have to fulfill several conditions in order to be used as scaffolds for this application. Among others, a suitable degradation rate, mechanical properties close to the native tissue and absence of cytotoxicity are required [9]. Poly(lactic acid) (PLA) fulfills these requirements and has also been approved by the Food and Drug Administration (FDA) as a material for several biomedical applications. Even though PLA has already been employed extensively for 3D scaffolding, the use of this biodegradable polymer has been scarcely reported for nozzle-based deposition methods $[13,14]$ due to poor resolution. PLA mechanical resistance and degradation rate are influenced by its crystallinity. Crystallites present in semicrystalline PLA are zones of high polymer density that reinforce the material, because of a much slower degradation compared to amorphous regions $[15,16]$. Therefore, scaffold mechanical properties and degradation rate could be tuned by controlling PLA crystallinity.

Nowadays, the production of scaffolds using nozzle-based systems usually consists in stacking struts of the selected material on top of each other to reach a final $3 D$ object $[6,8,10,17,18]$. Many parameters related to these struts and the resulting mesh can be varied such as strut diameter, space between them and orientation from one layer to another. However, more complex mesh designs are of great interest, especially in the field of tissue engineering. The gyroid morphology (Figure 1a) is a 
mesh composed of units made of quadruple junction points [19]. The branches have a curved shape and link other nodes throughout the structure. This kind of mesh provides interesting features for tissue scaffolding such as mechanical energy absorption and robustness, as well as good nutrient and waste diffusion owing to the high porosity and the minimal surface area [20, 21]. Gyroids for bone implantation were already printed in titanium or stainless steel by Electron Beam Melting (EBM) or Selective Laser Melting (SLM), two SFF methods $[20,22,23]$. However, these materials are heavy, nondegradable, and not suitable for all applications. Moreover, these SFF techniques are expensive. Digital Light Printing, a photocrosslinking-based method, has also been used with success to produce gyroids for biomedical applications [24]. Gaining approval and reaching the clinics by producing photopolymerizable resins containing nontoxic photoinitiators, solvents and other additives remains however challenging. In contrast, FDM is low cost, easy to implement and allows the use of several available medical grade thermoplastics, which could notably be biodegradable or not depending on the requirements. FDM has not been much considered for tissue engineering applications yet, probably because of its poor resolution compared to other additive manufacturing techniques. It has, to our knowledge, not yet been used for small and complex PLA model construction in the field of tissue engineering.

The aim of this work is to optimize the printing parameters of a PLA-based complex object at a scale close to the typical resolution limit of fused deposition modeling. The gyroid design will be used as a mesh for the 3D printing of scaffolds in reason of its remarkable properties, including large pore size, high interconnectivity, high porous volume and isotropy. The effect of mesh design, polymer crystallinity, and printing layer resolution on mechanical properties and degradation rate will also be assessed 


\section{Materials and methods}

\section{1. $3 D$ models}

The gyroid 3D model was downloaded from the 3DVIA website (Internet reference 1) and modified with an open source software (Blender V2.69.0, Blender Foundation, The Netherlands). The whole structure was made four times thicker to enable its printing with a common fused deposition modelling apparatus. Control models were also designed to produce scaffolds made of struts stacked on top of each other (Figure $1 \mathrm{~b}$ ), as often done in the literature related to 3D-printed scaffolds $[6,8,10,17,18]$. These models were drawn with the 123D Design software (Autodesk, V1.6) in order to have similar strut diameter and similar porous fraction as with the gyroid model.

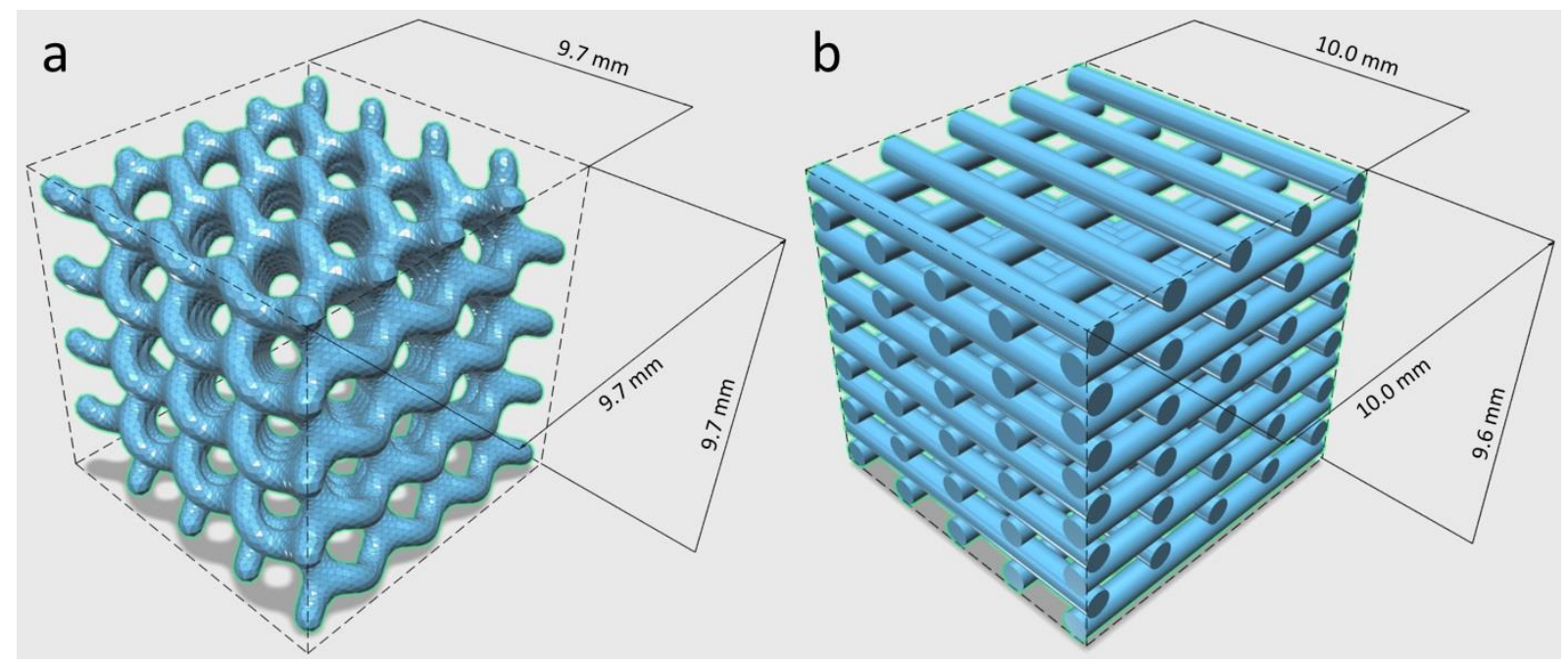

Figure 1: Gyroid 3D model (a) and control structure (strut-based scaffold) (b).

\subsection{Materials and printing conditions}

Polymers were printed by fused deposition modelling with a Makerbot Replicator 2X 3D printer (Makerbot, Germany) equipped with a dual extruder. Scaffolds were printed in poly(lactic acid) (PLA) (Makerbot, Germany), and High Impact polystyrene (HIPS) (Makerbot, Germany) was used as support material for printing optimization. Supports are polymer parts printed simultaneously with the structure, which carry PLA layers not supported by a layer already present underneath and would thus 
be deposited on void; the support material is then removed after printing. PLA blends from Makerbot are made from pellets of NatureWorks polylactide resin 4043D (NatureWorks LLC, USA). Numberaveraged molar mass $\left(M_{n}\right)$ and dispersity $(\Theta)$ values were previously determined with gel permeation chromatography using light scattering and were $57.0 \mathrm{~kg} / \mathrm{mol}$ and 2.43 , respectively [25]. Before printing, PLA was dried overnight at $80^{\circ} \mathrm{C}$, resulting in a change of its crystallinity (see more details in section XX). HIPS was removed after printing by dissolution in (R)-+-Limonene (VWR, USA). Then, the use of water-soluble poly(vinyl alcohol) (PVA) (Formfutura, the Netherlands) as a support material was evaluated to avoid the immersion of the scaffold in an organic solvent, with a view to better fit the requirements for clinical use.

Once modifications were made (see 2.1), the 3D model was imported in the MakerWare software (V. 2.4.1.24) and was duplicated to enable the printing of nine objects in parallel. Custom parameters were applied to program these structures with a set of printer path instructions. For all samples, PLA was printed with a concentric pattern, and the infill density, representing the fraction of void filled with polymer inside the pillars, was set to 1 . Gyroid samples (Figure $1 \mathrm{~A}$ ) were printed with a Z resolution of $100 \mu \mathrm{m}$ and with a nozzle diameter of $400 \mu \mathrm{m}$. Temperatures applied for the printing of PLA, HIPS and PVA were $220^{\circ} \mathrm{C}, 250^{\circ} \mathrm{C}$ and $230^{\circ} \mathrm{C}$, respectively. The support was printed $0.4 \mathrm{~mm}$ away from the structure, and its density, defined as the fraction of void filled with supporting polymer, was set to 0.5 . Purge walls were printed at the sides of the printed structures to get rid of overheated polymer and to sweep the nozzles. After printing, the supporting polymer (HIPS or PVA) was dissolved in limonene or water, respectively, in 3 baths of $8 \mathrm{~h}$ each. The need of a supporting material was further investigated. Supported and non-supported gyroid structures were compared. For samples without support, the set of printer path instructions was exactly the same as for the one with PVA support, but no filament was loaded into the second head of the printer.

Control samples (Figure 1B) were printed without support and the flow rate through the extrusion head was optimized to get the same void fraction as for the gyroid samples. These controls were 
printed with two different $Z$ resolutions: $100 \mu \mathrm{m}$ and $400 \mu \mathrm{m}$ (named control $100 \mu \mathrm{m}$ and control 400 $\mu \mathrm{m}$, respectively, hereafter), in order to analyze the effects of the printing resolution.

\section{3. $\mu C T$ scan}

HIPS-supported PLA scaffolds, treated with limonene to remove HIPS, were 3D imaged using a Phoenix NanoTom X-ray computed tomography equipment (General Electric Company, Fairfield, USA). This allowed the complex 3D scaffold structure to be imaged without destructive effects. The applied voltage and current were $40 \mathrm{kV}$ and $620 \mu \mathrm{A}$ respectively, with a molybdenum target. The exposure time was $500 \mathrm{~ms}$ and a frame averaging of 3 and image skip of 1 were applied. The obtained voxel size was $6.1 \mu \mathrm{m}^{3}$. The stack of scan images was then thresholded with CTVox software (Bruker microCT, Kontich, Belgium) to differentiate the polymer from the pores. The porous fraction was determined with the ImageJ 1.47v software (Wayne Rasband, National Institutes of Health, USA) [26]. Images were imported, cropped at the edges of the sample, thresholded into a binary stack and analyzed with the Voxel Counter plugin in ImageJ. Taking into account the distance between two images from the stack, the program evaluates the presence or absence of polymer voxel by voxel and sums up the information into a volume fraction.

\subsection{Porous volume measurement}

Gyroid structure porous fraction was measured experimentally on samples printed with a PVA support that was further eliminated with water. Mass and dimensions (width, W, length, L, and height, H) of gyroid and control structures were measured on 27 objects printed in three different batches (nine objects per batch). The porous fraction was computed with the following equation:

$$
\text { Porous fraction }[\%]=\frac{V_{o b j}\left[m^{3}\right]-V_{P L A}\left[m^{3}\right]}{V_{o b j}\left[m^{3}\right]} \times 100
$$

Where:

$V_{o b j}$ represents the entire volume of the object, obtained by equation (2) 


$$
V_{o b j}\left[m^{3}\right]=W[m] x L[m] x H[m]
$$

$V_{P L A}$ represents the volume occupied by PLA and is obtained by equation (3)

$$
V_{P L A}\left[m^{3}\right]=\frac{m[k g]}{\rho_{P L A}\left[k g / m^{3}\right]}
$$

Where:

$m$ is the mass of the object;

$\rho_{P L A}$ is the density of PLA, equal to $1250 \mathrm{~kg} / \mathrm{m}^{3}$ [16].

These experimental values were compared to the theoretical porous fraction measured with Netfabb Basic (V5.1) on the models used for printing.

\subsection{Surface topography}

PVA-supported PLA structures, treated with water to remove PVA, were analyzed with a Dektak XT (Bruker, USA) profilometer equipped with a $0.7 \mu \mathrm{m}$ diameter needle and using the Vision64 acquisition software (V5.40). As the surface at the edges of the samples was analyzed, the gyroid structure was filled almost entirely with a large cube to prevent the needle of the profilometer to be stuck in a pore (Figure 3). Three different sides of each sample $(\mathrm{N}=3$ ) were scanned with a resolution of $0.075 \mu \mathrm{m} / \mathrm{pt}$ (vertical resolution) and $12 \mu \mathrm{m} /$ line (horizontal resolution). The force applied on the needle was 0.06 $\mathrm{mg}$ (sides) and $0.5 \mathrm{mg}$ (top).

\subsection{Crystallinity fraction evaluation}

PVA-supported PLA gyroid structures, treated with water to remove PVA, and PLA control samples were annealed overnight at $80^{\circ} \mathrm{C}$. Crystallinity, glass transition temperature $\left(T_{\mathrm{g}}\right)$ and change in specific heat capacity $\left(\Delta C_{p}\right)$ at the $T_{g}$ of annealed and not annealed printed samples were measured by differential scanning calorimetry (Mettler Toledo DSC821, Belgium). Calibration was done with Indium. Samples were cut to obtain fragments with a mass ranging from 8 to $11 \mathrm{mg}$ ( $\mathrm{n}=3$ per condition) and 
loaded into $40 \mu$ l aluminum pans. For $T_{g}$ measurement and $\Delta C_{p}$ at this $T_{g}$, a temperature ramp was applied from $20^{\circ} \mathrm{C}$ to $250^{\circ} \mathrm{C}$ at a rate of $10^{\circ} \mathrm{C} / \mathrm{min}$. The samples were then cooled at a rate of $10^{\circ} \mathrm{C} / \mathrm{min}$, and a second cycle was used as polymer state control. For crystallinity measurement, the heating ramp was increased to $100^{\circ} \mathrm{C} / \mathrm{min}$ in order to minimize sample re-crystallization inside the chamber. Percentage of crystallinity was computed based on the linear increase of enthalpy of fusion with crystallinity, and using a reference heat of enthalpy of $0 \mathrm{~J} / \mathrm{g}$ and $93.1 \mathrm{~J} / \mathrm{g}$ for theoretically $0 \%$ and $100 \%$ crystalline PLA, respectively.

\subsection{Compression tests}

Compression tests were performed (LRX plus, Lloyd Instruments) on both amorphous and crystalline gyroids $(n=3)$, control $400 \mu m(n=3)$ and control $100 \mu m(n=3)$ structures to evaluate the effect of mesh geometry, crystallinity and printing resolution on scaffold mechanical properties. The speed used for the compression test was $1 \mathrm{~mm} / \mathrm{min}$. Each sample was placed between two parallel steel plates along two axes: perpendicularly and parallel to the printed layers of the sample. Force and displacement were recorded and converted into stress and strain, respectively, taking into account scaffold dimensions. The square coming from the horizontal cross section of the imaginary cube containing the scaffold was chosen as the area needed for the conversion of force into strain. Apparent compression moduli were obtained from the slope of the linear region of the sigmoid (slope at the initial linear part of stress-strain curve) and yield strengths were extracted using the offset method with a value of $1 \%$ (see Figure SI2 in Supporting Information for details).

\subsection{Scaffold in vitro degradation}

Amorphous $(n=4)$ and annealed $(n=4)$ gyroid, control $100 \mu \mathrm{m}$ and control $400 \mu \mathrm{m}$ structures were weighed. They were then disinfected in ethanol 70\%, dried and placed in $5 \mathrm{ml}$ of phosphate buffered saline (PBS) containing $0.5 \%$ of $\mathrm{NaN}_{3}$ to avoid development of microorganisms. Samples were incubated at $37^{\circ} \mathrm{C}$ and each week, half of the degradation medium was replaced by fresh PBS containing $\mathrm{NaN}_{3}$. Each month, samples were dehydrated in ethanol and dried at $37^{\circ} \mathrm{C}$ during $6 \mathrm{~h}$ and 
then weighed. Only the main parts of samples (pieces bigger than 1 strut) were taken into account. Again, samples were disinfected in ethanol 70\%, rinsed twice in PBS and once in PBS containing 0.5\% of $\mathrm{NaN}_{3}$ before placing them back in their own degradation medium ( $5 \mathrm{ml}$ PBS containing $0.5 \%$ of $\mathrm{NaN}_{3}$ ). The study was carried out until the main body of the scaffold fell apart. At week 64 , pictures of the samples were taken with a low magnification microscope, at the same magnification (12X) and with the same backlight intensity.

\subsection{Statistical analysis}

ANOVA and Tukey's HSD were performed using JMP ${ }^{\circ}$, Version 12 Pro. (SAS Institute Inc., USA) ( $\left.\alpha=0.05\right)$. 


\section{Results and discussion}

\subsection{D scanning of printed gyroid structures}

The HIPS-supported gyroid structure was scanned by $\mu \mathrm{CT}$ after HIPS dissolution. Figures 2.a and 2.b show the model and the printed gyroid scan, respectively, both cropped at the front and the top of the structure for the sake of clarity. The three-dimensional reconstruction matches the computerized model very well, and nodes as well as gyrating junctions are well defined and clearly visible. Besides, the $\mu \mathrm{CT}$ scan shows large pores that are completely interconnected. Cross-sections can be extracted and isolated from the 3D in silico model and from the $\mu \mathrm{CT}$ scan. Figure 2.d presents a printed slice of the sample, which is in close agreement with the slice coming from the model (Figure 2.c). This crosssection shows that the bean-shaped units, corresponding to the pillars of the gyroid structure, are similar to the model. Besides a good nutrient and waste diffusion, the interconnection and the large pore size (pore projections of $800 \mu \mathrm{m}$ ) should allow a good cell-cell communication and favor tissue ingrowth. As pore projections are at least 10 times larger than cells, this structure should allow cells to migrate, proliferate and differentiate within the scaffold, and vascularization could be initiated [11]. Geometrical scaffold properties such as pore size and shape and even local curvature were shown to have an impact on the control of cell proliferation and fate, both in vitro and in vivo [27]. Depending on pore size and scaffold dimensions, a dynamic culture environment (with perfusion reactors) is sometimes needed to increase mass transport of nutrient and oxygen [28]. Flow-associated shear stress linked to geometric features of the scaffold has been shown to influence neo-tissue growth kinetics. Small shear stress ( $0.015 \mathrm{~Pa}$ for human bone marrow MSCs) has a positive effect while higher shear stresses $(0.05 \mathrm{~Pa})$ decrease neo-tissue growth or result in inhomogeneous tissue-engineered constructs. The spring shape design of the gyroid shows a lot of curved surfaces and only rare spots of the structure would be perpendicular to the flow in the case of culture in dynamic conditions. Shear stress should therefore be reduced compared to a strut-based scaffold, and flow patterns should be more uniform. The printed gyroid structure is thus an appropriate scaffold for tissue reconstruction. 


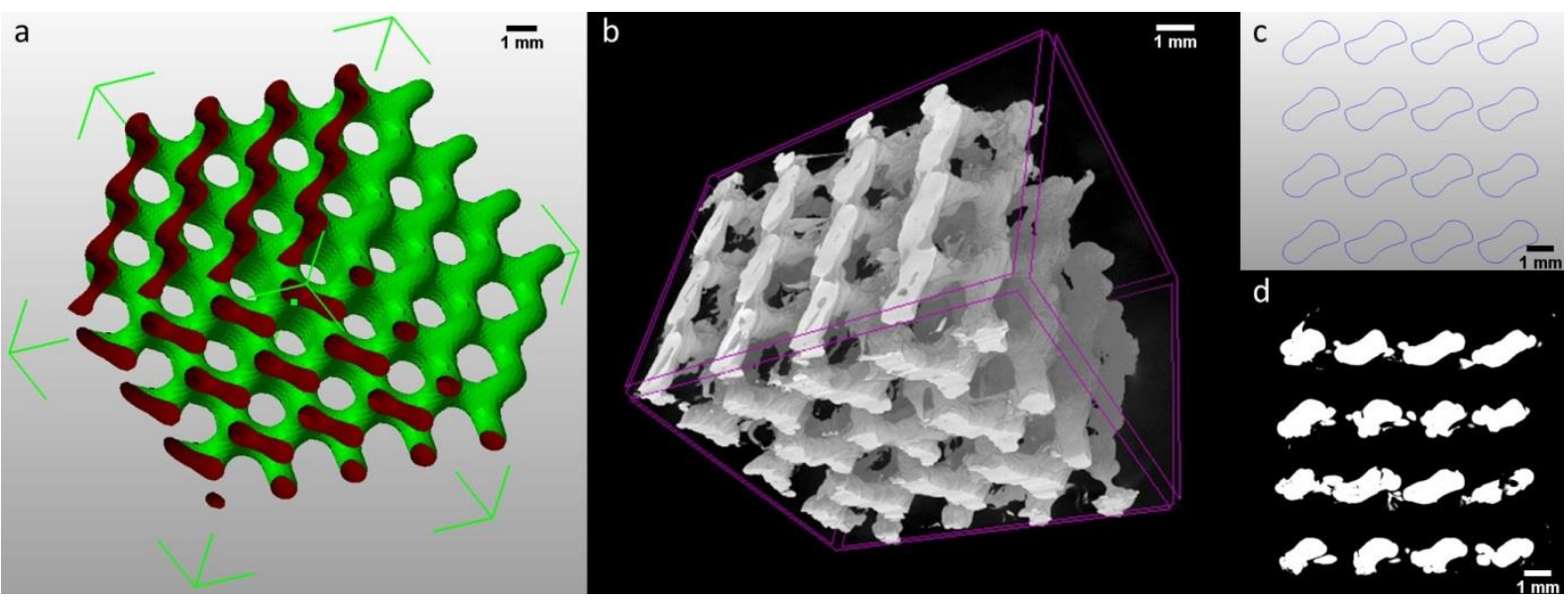

Figure 2: Model (a) and printed gyroid $\mu \mathrm{CT}$ scan (b) both cropped at the front and the top of the structure for the sake of visualization. Model (c) and $\mu \mathrm{CT}$ scan (d) of the corresponding printed cross-sections.

\subsection{Porosity of printed gyroids}

In complement to the 3D visualization of the gyroid structure, its porous fraction was determined, based on the mass and dimensions of the samples (see 2.4), and on $\mu \mathrm{CT}$ stack images (Table 1). Both values were compared to the porosity predicted theoretically according to the in silico model. In each case, porosity is in the range of $70-75 \%$. The similarity of experimental and theoretical porosity values, as well as the small standard deviation of experimental values based on mass and dimensions, emphasize the reliability of the gyroid model printing. Moreover void fraction is important as it will affect mechanical resistance.

Table 1: Printed gyroid porosity computed from mass and dimensions of gyroid structure ( $\mathrm{N}=27$; standard deviation; middle) and from $\mu \mathrm{CT}$ images processing ( $\mathrm{N}=1$; bottom), as compared to the theoretical value (top).

\section{Porosity (\%)}

Theoretical

Experimental - From mass and dimensions

Experimental - From $\mu \mathrm{CT}$ scan images
73.7

$70.8(0.5)$

74.7

\subsection{Profilometry of printed gyroid scaffolds}


Characterization was further conducted at the micrometer scale, which is of interest regarding cellmaterial interactions. Profilometry was used to analyze the surface at the edges of the sample. As represented in Figure 3, the top and two sides of printed cubes, representing different orientations of the junctions compared to the printed layer, were analyzed. Profilometry analysis shows the stacked polymer layers of around $100 \mu \mathrm{m}$ thick. The surface of these layers is, in each case, rather smooth at the micrometer scale. Despite the presence of the layering artifact, the 3D design is respected at the $100 \mu \mathrm{m}$-scale, as already evidenced by the $\mu \mathrm{CT}$ scan ( $\mathrm{N}=3$ ). Micro- and nano-topography on 2D surfaces has been shown to influence cell shape, morphology and in some cases proliferation. Cells from many different origins were shown to align parallel to micro-and nano-grooves and, in some cases, this even influenced their migration [29-31]. Here, cells could align parallel to the grooves created by the stacking of the layers. This layer artifact inherent to 3D printing techniques could be exploited to guide cell growth and migration.

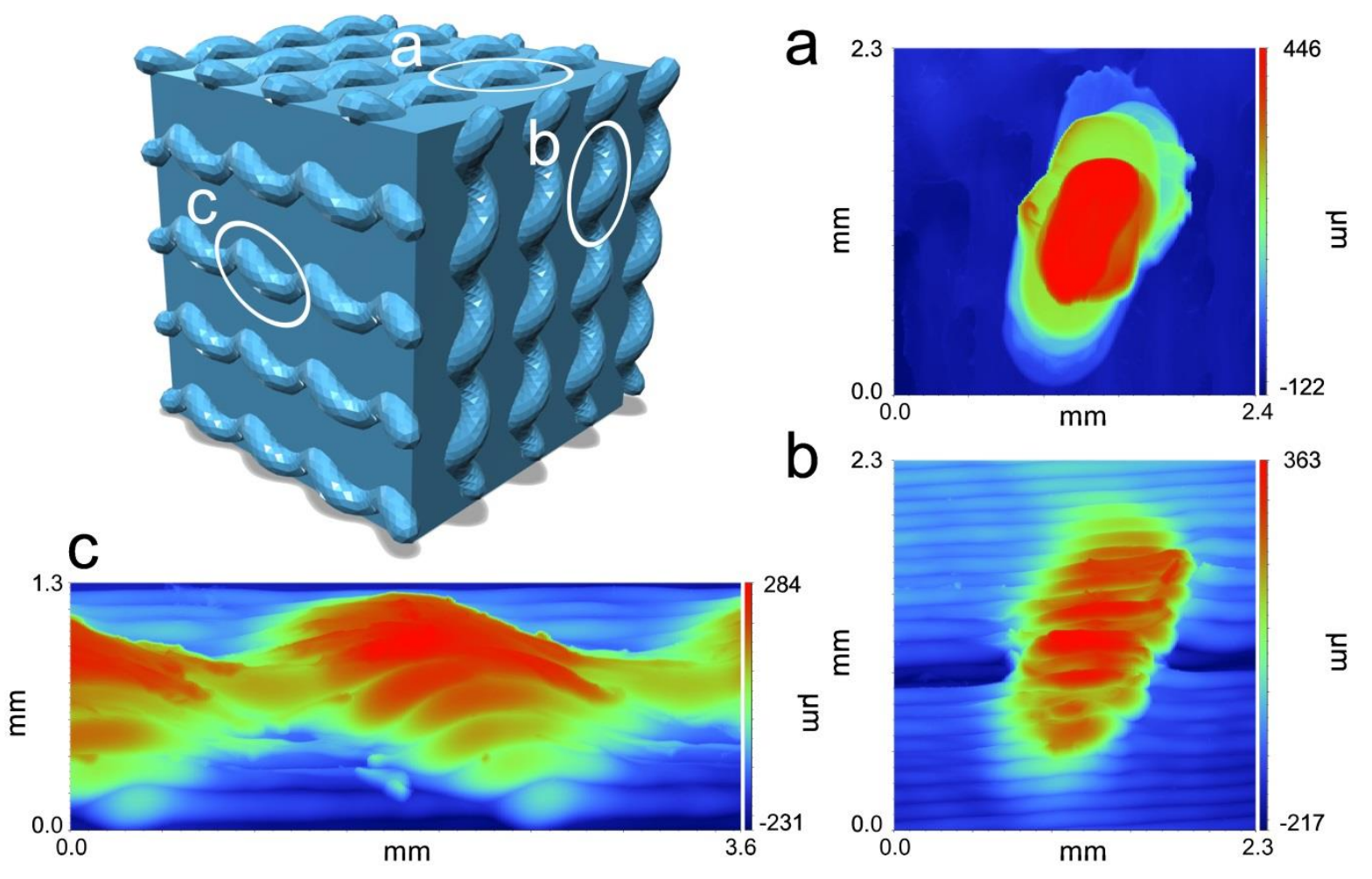

Figure 3: Simplified geometry for profilometry analysis (upper left) and topographic scans obtained by profilometry of circled areas on the top (a) and two different sides $(b, c)$ of the sample. 


\subsection{Influence of the support on shape fidelity}

The need of a supporting polymer to achieve good gyroid printing was investigated. Since most of the structure is self-supported, almost no overhang is present in the design, and all bridges are sufficiently small for the printer to overcome printing issues, printing without support should be possible and would constitute an interesting alternative since a dissolution step would not be required to eliminate the supporting material. Gyroids were printed with and without the addition of PVA in the second nozzle. Scaffold morphology was significantly impacted by the absence or presence of a supporting structure during printing, as it can be observed in Figure 4 where black arrows point out the presence of polymer leaking out of the nozzle, known as oozing, on the non-supported samples and their absence on the supported ones. In addition, the support improves the deposition of the layers with straight orientation instead of wavy-like structure (grey arrows) and leads to less polymer drops (white arrows). The support improves the printing quality, even though the structure can be printed without support. The absence of support results in a decrease of shape accuracy.
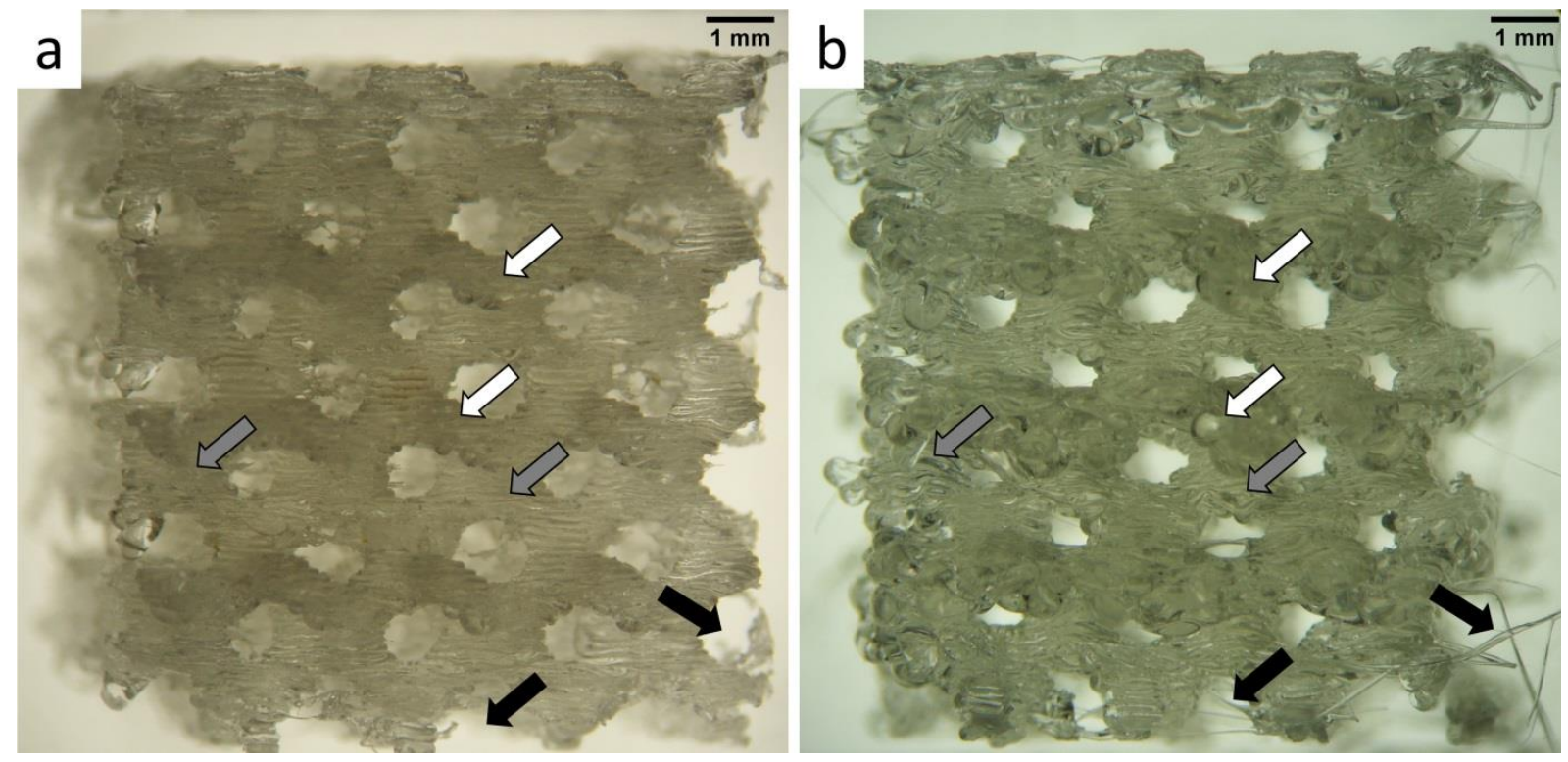

Figure 4: Pictures of gyroid structures printed with (a) and without (b) PVA support (that was later dissolved in water). Black, grey and white arrows show the presence or absence of stringing, wavy like layers and polymer drops, respectively.

\subsection{Impact of annealing on printed PLA}


The crystallinity of printed samples annealed overnight at $80^{\circ} \mathrm{C}$ was compared to the crystallinity of the as-printed polymer, using DSC (Table 2). For all samples, $T_{g}$ values were close to each other and to the theoretical value of around $58^{\circ} \mathrm{C}[16]$. The structures, regardless of the design, came out of the printer in an amorphous state (crystallinity below detection level). The absence of crystallinity for notannealed samples can be easily explained by a quick cooling of the polymer once deposited by the nozzle after being melted at $220^{\circ} \mathrm{C}$ (melting point around $150^{\circ} \mathrm{C}$ ). The polymer does not have time to crystallize before reaching its $\mathrm{T}_{\mathrm{g}}$. Sample annealing overnight at $80^{\circ} \mathrm{C}$ is sufficient to reach a crystallinity of $37 \%$ for the gyroid structure, and of $40 \%$ for the control structures. Upon annealing, the polymer is kept at a temperature above the $T_{g}$ during a period of time sufficient for the chains to rearrange in small crystalline zones and form spherulites. These results are consistent with the measurement of $\Delta C_{p}$, ie the energy required to turn the state of the amorphous part of a polymer from vitreous to viscous, which is around $0.53 \mathrm{~J} \cdot \mathrm{g}^{-1} \cdot \mathrm{K}^{-1}$ for the amorphous samples compared to $0.22 \mathrm{~J} \cdot \mathrm{g}^{-1} \cdot \mathrm{K}^{-1}$ for the annealed ones. This annealing process, performed after printing the structures, can be used to modify the crystallinity of the scaffold and hence, to tune its mechanical properties and its degradation rate.

Table 2: DSC measurement of glass transition temperature $\left(T_{g}\right)$, change in specific heat capacity $\left(\Delta C_{p}\right)$ and degree of crystallinity for not-annealed and annealed gyroid and control samples. Mean (standard deviation); BDL = below detection $\operatorname{limit}(\mathbf{N}=3)$

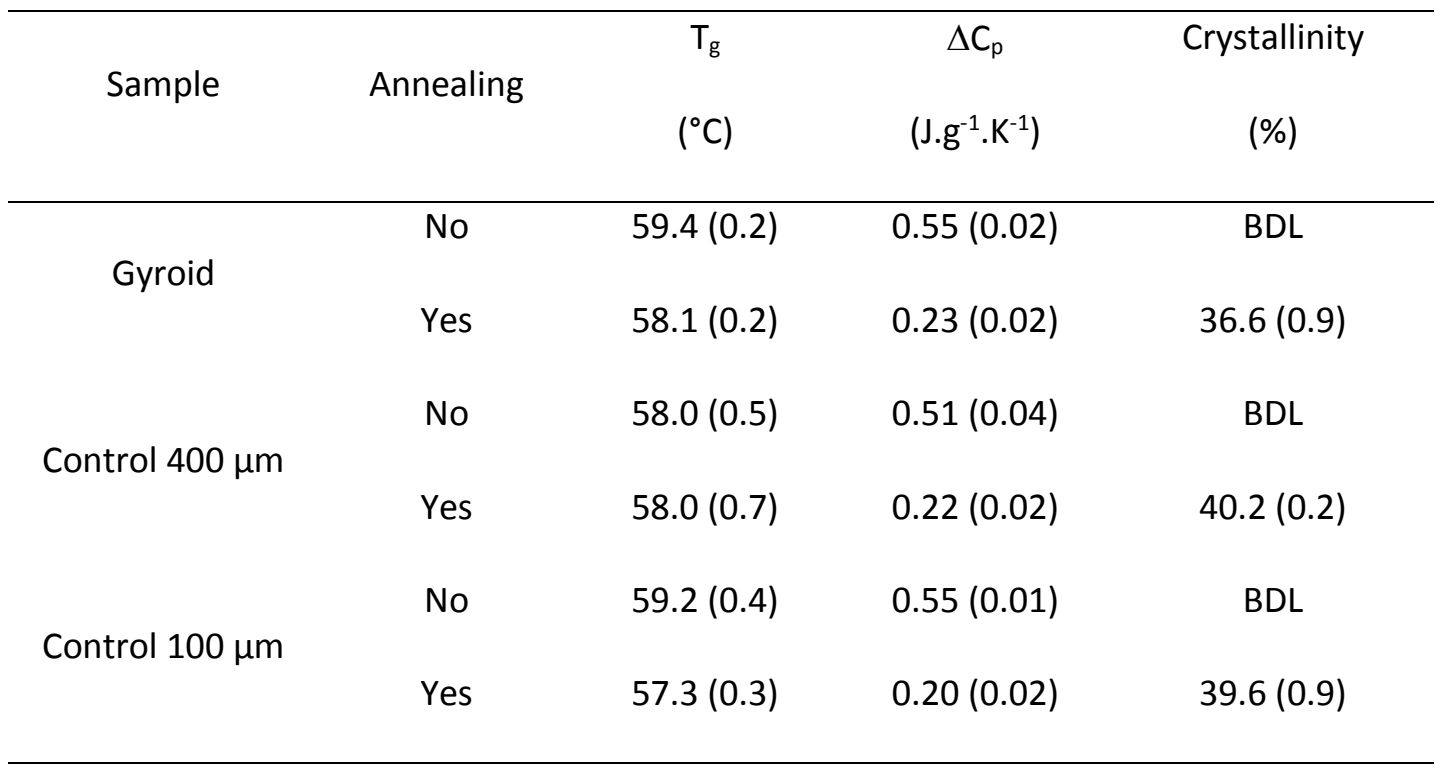


The gyroid structure was chosen for its spring shape design that should provide isotropic resistance to pressure and high elasticity. Mechanical tests were performed to study the behavior of the structure depending on (i) the direction of compression with respect to the printed layers, (ii) printing resolution, and (iii) polymer crystallinity. All these parameters are expected to influence the mechanical properties of a scaffold [32-34]. Figure 5 presents a typical stress-strain curve for each type of sample and in each direction of compression (force applied parallel or perpendicular to the printed layers). Stress-strain curves can be compared for the different conditions as the nature of the polymer and the porosity were kept the same. For the crystalline and amorphous gyroid structures, the curves are similar whatever the compression direction. In contrast, the compression direction significantly impacts the behavior of control scaffolds. When the compression load is applied parallel to the printed layers, peaks appear in the graphs. This phenomenon is due to buckling of the columns of the structures, one level after the other, in the process of compression. The crystallinity and the thickness of the printed layers do affect this buckling behavior. Yield strengths were determined (see Supporting Information, Figure SI3) and they are significantly higher for crystalline samples and for thicker printed layers (400 $\mu \mathrm{m}$ versus $100 \mu \mathrm{m})$. This means that more pressure is needed to allow the buckling of the columns for crystalline compared to amorphous samples, as well as for thicker compared to thinner printed layers. This was expected since buckling is a phenomenon of elastic instability, which happens at lower loads when the bending stiffness of a loaded column decreases. Bending stiffness is proportional to the elastic modulus, which will be higher for the crystalline phase. On the other hand, thinner printed layers create more weak boundaries within each column, which may decrease bending stiffness.

Slopes were extracted from the linear part of the stress-strain curves, to give an apparent compression modulus (see Supporting Information, Figure SI3). The results show that the gyroid structure, with an apparent compression modulus of about $50 \mathrm{MPa}$, has a more deformable behavior owing to its spring shape. Besides, layer thickness has an effect only if the load is applied parallel to 
the printed layers. The crystallinity, surprisingly, does not have much influence on the apparent compression modulus.

These results reveal a clear effect of the mesh geometry, the printed layer thickness and the crystallinity on the resistance to compression. The gyroid sample shows a more deformable and isotropic behavior when compressed compared to both control structures. This isotropy is of high interest in tissue engineering as it would allow the printing and the implantation of scaffolds that would support the pressure coming from any direction equally, regardless of the printing or implant orientation.

Gyroid geometry was studied in the case of Solid Freeform Fabrication of metals (titanium alloys) [35]. Reducing the angle between the struts and the axial compression direction (changing the eccentricity of pore projections) in a gyroid increased elastic moduli and compressive strength, while angle values over $45^{\circ}$ decreased both of them [22]. Nevertheless, it would be reasonable to expect that the isotropic behavior regarding compression will be lost if angles diverge from $45^{\circ}$. Mechanical testing of titanium alloy gyroids was compared to octahedron models and displayed a higher yield and plateau stress [36]. A superior fatigue resistance was also emphasized and could be attributed to the continuous rate of curvature of gyroids, removing nodal points which may favors cracks initiations. However, all these compression studies were realized along only one axis. As shown by Heinl et al., loads applied in other directions can strongly modify the mechanical behavior of the structure [32]. They compared a gyroid and a strut-based design printed in titanium alloy. The difference in porosity makes comparison between the structures meaningless. However, both gyroid and strut-based structure showed an elastic modulus 2 and 3 times lower, respectively, for a compression parallel to printed layers if compared with a perpendicular compression. This is, however, quite inconsistent with our result, as in our case the lower moduli were observed for a compression perpendicular to printed layers. This could be due to the difference in the model used (size of struts, space between struts,...) or the difference in product manufacture (electron beam melting versus fused deposition modeling). 


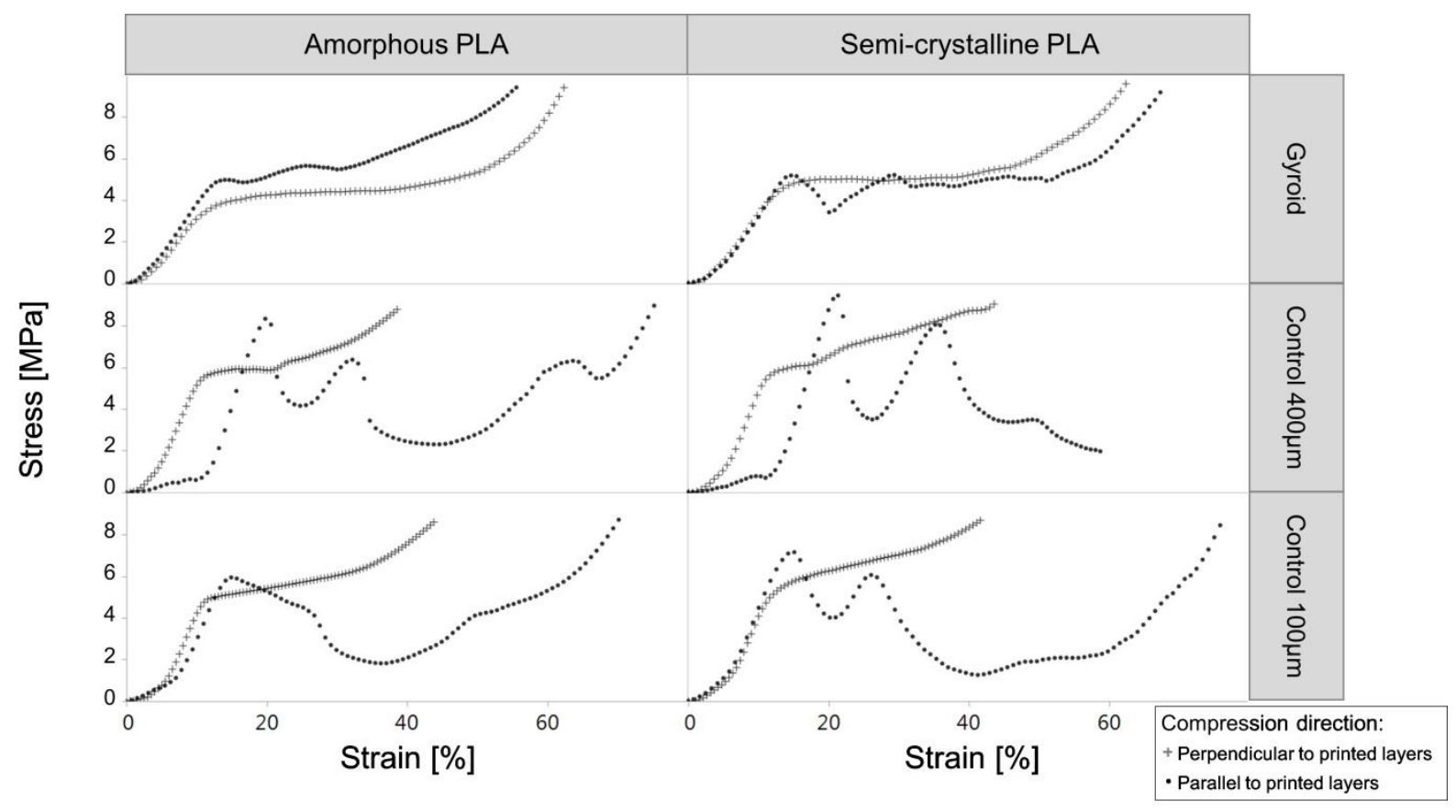

Figure 5 : Stress-strain curves of amorphous (left) and semi-crystalline (right) gyroid (top) and control samples (middle and bottom) along two axes: perpendicular $(+)$ and parallel $(\bullet)$ to printed layers. Results were obtained in triplicate but a single representative result is shown for the sake of clarity. All curves are available in Supporting Information (Figure SI1)

\subsection{Impact of geometry, printing resolution and crystallinity of the structures on in vitro degradation} kinetics

PLA mainly degrades by ester linkage hydrolysis, which occurs in presence of water and is catalyzed in acidic or basic conditions. Gyroid and control samples, in amorphous and semi-crystalline state, were immersed in phosphate buffered saline (PBS) during 84 weeks.

The mass of the samples was stable during about 50 weeks (Figure 6). From week 52, samples started to drastically degrade. The semi-crystalline control $100 \mu \mathrm{m}$, amorphous gyroid and amorphous control $100 \mu \mathrm{m}$ were the most degraded at week 84 , with a remaining mass of 20,43 and $46 \%$ of the initial mass, respectively. The relative masses after 84 weeks were significantly higher for the $400 \mu \mathrm{m}$ resolution samples ( $80 \%$ for amorphous and $73 \%$ for crystalline samples), meaning that thicker printed layers slow down the degradation process. Concerning the crystallinity, the effect seems less 
obvious. Indeed, annealing seems to weaken $400 \mu \mathrm{m}$ and $100 \mu \mathrm{m}$ control samples, but not the gyroid samples.

This sudden degradation is well known for PLA and described in literature. As water is able to penetrate the PLA bulk, hydrolysis happens throughout the entire polymer matrix. This phenomenon is called "bulk degradation" and it has to be distinguished from "surface erosion" which happens in more hydrophobic polymers such as polycaprolactone. The bulk degradation of PLA is also the result of an autocatalytic process. When an ester bond is cleaved, a new carboxylic acid function is generated, the $\mathrm{pH}$ in the bulk decreases, and the degradation kinetic increases [16, 37].

Felfel et al. and Serra et al. did not observe significant degradation of PLA films after 8 weeks at $37^{\circ} \mathrm{C}$ in PBS and simulated body fluid, respectively [13,38]. Höglund et al. obtained a $75 \%$ relative mass for polylactide 3051D (NatureWorks LLC, USA) and $85 \%$ for $250 \mu \mathrm{m}$-thick PLLA films after 26 weeks of degradation at $37^{\circ} \mathrm{C}$ in PBS [39]. Ravi et al. worked on 3D printed porous sheets ( $800 \mu \mathrm{m}$ thick) and measured relative masses between 76 and $89 \%$ after 26 weeks for the same polymer (PLLA, PURAC, The Netherlands) for distinct pore designs [40]. Both studies showed higher degradation rates compared to our study. However, both PLA source and sample geometry and dimensions were different. Indeed, several factors influence PLA degradation rate including the morphology, degree of crystallinity, molar mass, hydrophobicity, size and geometry of the samples, stereocomplex formation, and environmental conditions ( $\mathrm{pH}$ and temperature)[39]. In some cases, PLA can take more than 3 years for a complete degradation [38]. These differences in degradation time emphasize the importance of extended degradation studies on real or close-to-real sample sizes and geometries. 


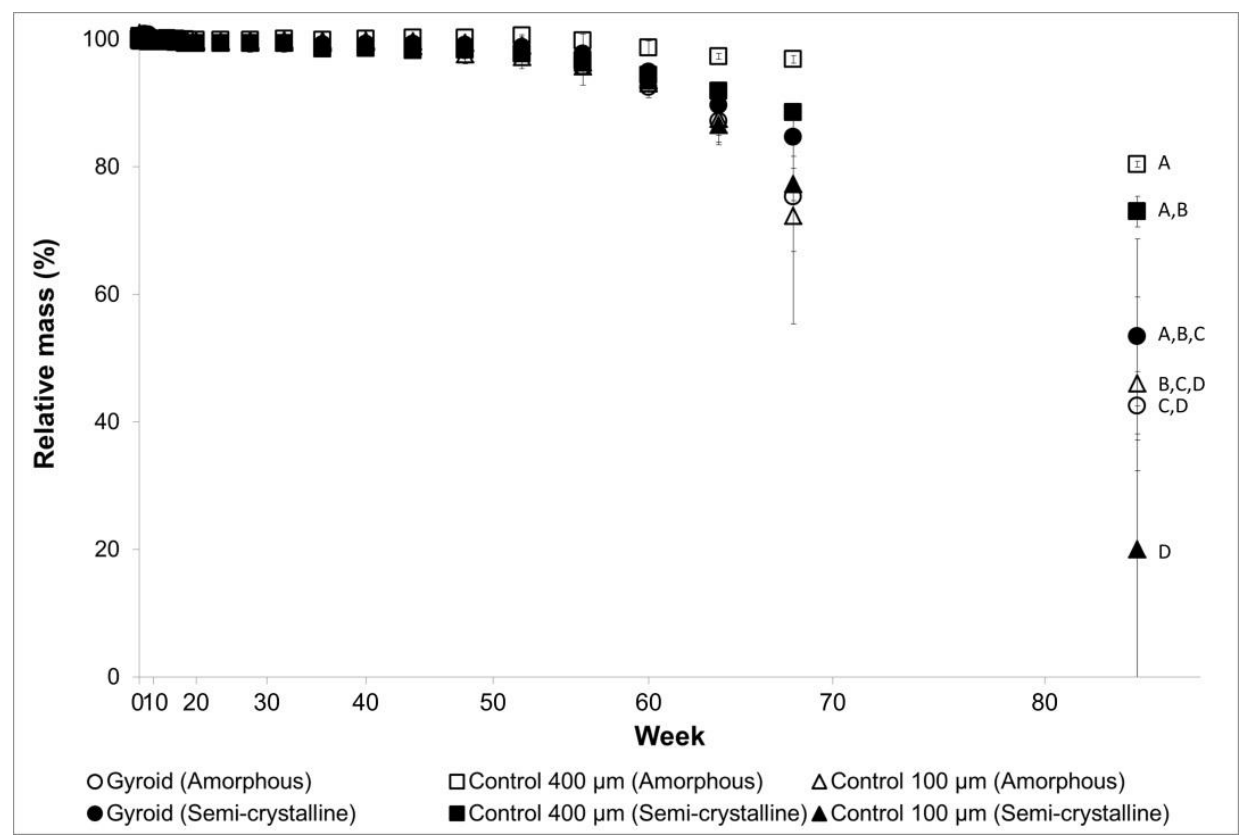

Figure 6: Degradation of not-annealed and annealed gyroid and control samples in phosphate buffered saline (PBS). For the sake of clarity, a non-linear $\mathrm{x}$ axis was used (error bar= standard deviation, $\mathrm{N}=4$ ). Values not sharing the same letter are significantly different $(\alpha=0.05)$.

Photographs of the degraded structures were taken at week 64 (Figure 7). Although sample masses were stable for 50 weeks, macroscopic changes were visible for amorphous scaffolds: a change in color, from clear and translucent to a milky white and completely opaque polymer, was already visible after 3 weeks. This has already been attributed to the swelling of PLA at an early stage of the bulk erosion process [40] which leads to a change in optical properties. In contrast, such change was not observed for crystalline samples, which were less translucent initially.

Scaffold geometry also impacts degradation. Control scaffolds degrade by losing struts one after the other, whereas the gyroid structure tends to retain its integrity. This could be related to the inclination angles of the circular gyroid struts that continuously vary all over the structure. This results in slight changes in area and position between two adjacent layers [20]. The layers are then more interconnected in the case of the gyroid compared to the strut-based scaffold, which has only a few connection points between struts of different levels. However, in the case of the amorphous gyroid, cracks arise at the junctions of printed layers, resulting in elongation of the sample. Therefore, the 
annealing process seems to have a positive effect on the cohesion of the printed layers of the gyroid samples.

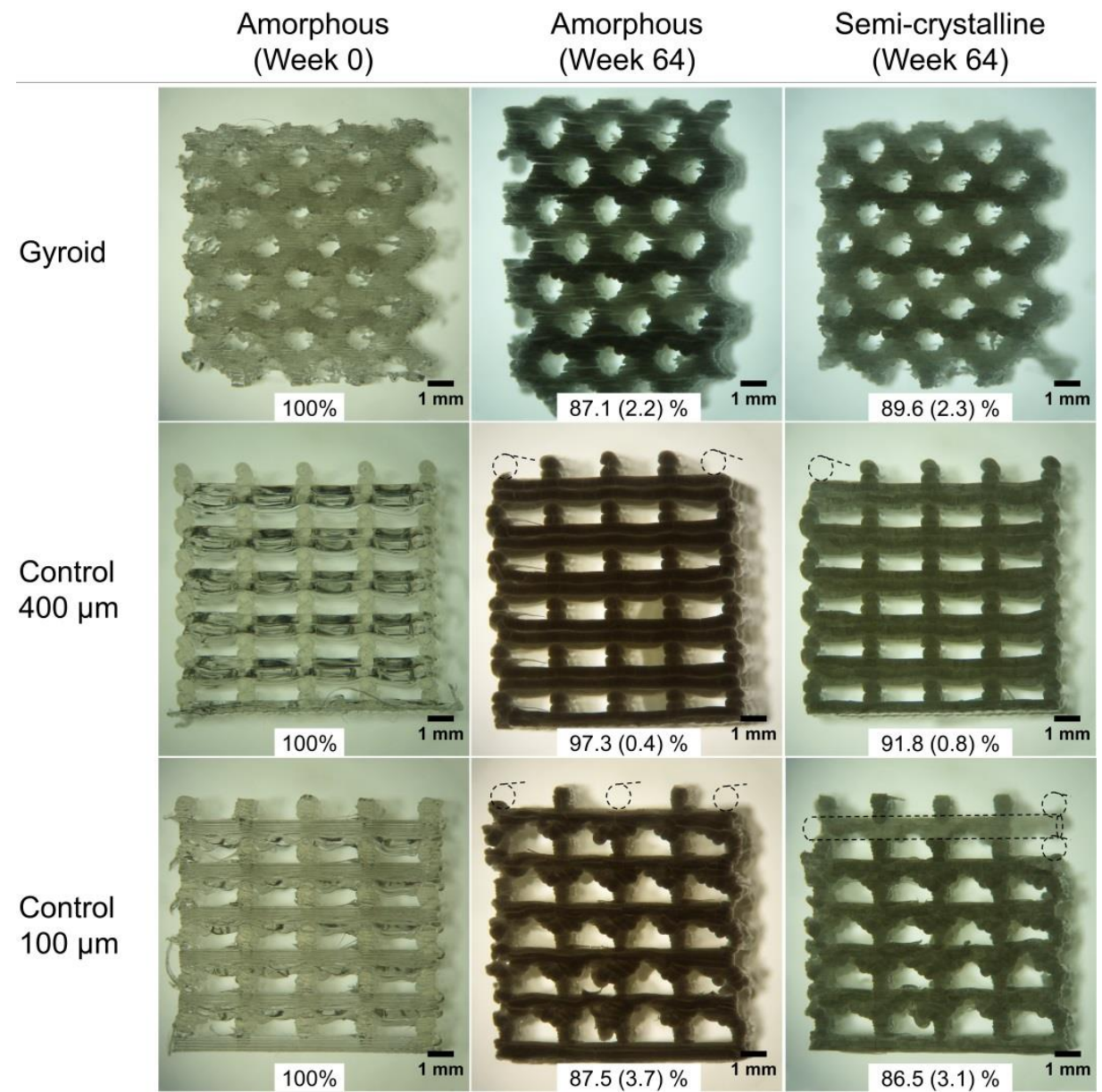

Figure 7: Pictures of gyroid (top) and control (middle $-400 \mu \mathrm{m}$ and bottom $-100 \mu \mathrm{m}$ ) samples, as produced (left) or after 64 weeks of degradation (middle - amorphous samples, right - semi-crystalline samples). Dotted lines represent struts present at the beginning of the experiment that were lost due to structure degradation. Means of relative masses and standard deviations $(n=4)$ are presented in white boxes.

The degradation seems affected only by printed layer thickness, a higher thickness reducing the speed of the process. Neither crystallinity nor mesh geometry affects significantly the degradation rate even though the change in mesh geometry will influence the detachment of pieces from the structures. Besides this, the annealing process improves layers attachment in the case of gyroid samples.

\section{Conclusions}


A complex gyroid scaffold was printed in PLA close to the resolution limit of fused deposition modelling, a simple and low-cost additive manufacturing technique. This geometry is particularly suitable for tissue engineering approaches given the large pore size, high porous volume and mechanical robustness. The reliability of the printing procedure was demonstrated based on a $\mu \mathrm{CT}$ scan, on porous volume measurement and on surface topography analysis. PLA is a FDA-approved biodegradable and implantable polymer. The water-soluble PVA support was shown to be important to obtain a controlled and well-defined shape of the gyroid structure, and it can be easily dissolved after printing. Annealing was used to increase the crystallinity fraction of the polymer, with a view to tune mechanical properties and degradation rate. Mechanical compression tests showed that crystallinity, layer resolution, and especially the structure design influence the stress-strain curves. Moreover, the gyroid structure presents an isotropic behavior regarding compression, in contrast with strut-based designs commonly reported in the literature. Degradation tests highlighted the faster degradation for thinner printed layers, whereas the gyroid geometry prevents the detachment of pieces from the structure upon degradation, and the annealing process increases layers attachment. Results, brought together, show that the gyroid design shows superior properties to be used for personalized implantable scaffolds for tissue engineering.

\section{Acknowledgements}

We sincerely thank Michel Sclavons and Prof. Sophie Demoustier-Champagne for helpful discussions and Bio- and Soft Matter unit (BSMA) for the access to the 3D printer as well as Julian Leprince for the access to the mechanical test instrument and Luc Randolph for his help in stress-strain curve analysis, Pascal Van Velthem for DSC training, Prof. Arnaud Delcorte for the access to the profilometer and Claude Poleunis for the training and Prof. Yves Dufrêne for the access to the low magnification microscope. The work was supported by the Interuniversity Attraction Pole Program (P07/05) and the Belgian National Foundation for Scientific Research (FNRS). Anne des Rieux is a F.R.S.-FNRS Research Associate. 


\section{References}

1. Verhulsel, M., et al., A review of microfabrication and hydrogel engineering for micro-organs on chips. Biomaterials, 2014. 35(6): p. 1816-1832.

2. Cox, S.C., et al., 3D printing of porous hydroxyapatite scaffolds intended for use in bone tissue engineering applications. Mater Sci Eng C Mater Biol Appl, 2015. 47: p. 237-47.

3. Pineda, E.T., R.M. Nerem, and T. Ahsan, Differentiation patterns of embryonic stem cells in two- versus three-dimensional culture. Cells Tissues Organs, 2013. 197(5): p. 399-410.

4. Tang, L., et al., Adipose-Derived Stem Cells Expressing the Neurogenin-2 Promote Functional Recovery After Spinal Cord Injury in Rat. Cellular and Molecular Neurobiology, 2016. 36(5): p. 657-667.

5. Gao, F., et al., Mesenchymal stem cells and immunomodulation: current status and future prospects. Cell Death Dis, 2016. 7: p. e2062.

6. Park, S., et al., 3D polycaprolactone scaffolds with controlled pore structure using a rapid prototyping system. J Mater Sci Mater Med, 2009. 20(1): p. 229-34.

7. Ahmed, T.A.E., E.V. Dare, and M. Hincke, Fibrin: A versatile scaffold for tissue engineering applications. Tissue Engineering - Part B: Reviews, 2008. 14(2): p. 199-215.

8. Lee, H., et al., Cell(MC3T3-E1)-printed poly(-caprolactone)/alginate hybrid scaffolds for tissue regeneration. Macromol Rapid Commun, 2013. 34(2): p. 142-9.

9. Jung, J.W., et al., Octahedron pore architecture to enhance flexibility of nasal implant-shaped scaffold for rhinoplasty. International Journal of Precision Engineering and Manufacturing, 2014. 15(12): p. 2611-2616.

10. Trachtenberg, J.E., et al., Open-source three-dimensional printing of biodegradable polymer scaffolds for tissue engineering. Journal of Biomedical Materials Research Part A, 2014: p. n/a-n/a.

11. Seyednejad, H., et al., In vivo biocompatibility and biodegradation of 3D-printed porous scaffolds based on a hydroxyl-functionalized poly(epsilon-caprolactone). Biomaterials, 2012. 33(17): p. 4309-18.

12. Gross, B.C., et al., Evaluation of 3D printing and its potential impact on biotechnology and the chemical sciences. Anal Chem, 2014. 86(7): p. 3240-53.

13. Serra, T., et al., Relevance of PEG in PLA-based blends for tissue engineering 3D-printed scaffolds. Mater Sci Eng C Mater Biol Appl, 2014. 38: p. 55-62.

14. Serra, T., J.A. Planell, and M. Navarro, High-resolution PLA-based composite scaffolds via 3-D printing technology. Acta Biomater, 2013. 9(3): p. 5521-30.

15. Uzel, A.P., et al., Preclinical and clinical cases of new absorbable composite interference screws in osteoarticular surgery, in Key Engineering Materials. 2013. p. 325-330.

16. Henton, D.E., et al., Polylactic Acid Technology, in Natural Fibers, Biopolymers, and Biocomposites, T.F. Group, Editor. 2005, CRC Press. p. 527-577.

17. Temple, J.P., et al., Engineering anatomically shaped vascularized bone grafts with hASCs and 3D-printed PCL scaffolds. J Biomed Mater Res A, 2014. 102(12): p. 4317-25.

18. Wang, F., et al. Fabrication of cellular poly-e-caprolactone (PCL) scaffolds by precision extruding deposition process. in Bioengineering, Proceedings of the Northeast Conference. 2003.

19. Dolan, J.A., et al., Optical Properties of Gyroid Structured Materials: From Photonic Crystals to Metamaterials. Advanced Optical Materials, 2015. 3(1): p. 12-32.

20. Yan, C., et al., Evaluations of cellular lattice structures manufactured using selective laser melting. International Journal of Machine Tools and Manufacture, 2012. 62: p. 32-38. 
21. Torres-Rendon, J.G., et al., Bioactive gyroid scaffolds formed by sacrificial templating of nanocellulose and nanochitin hydrogels as instructive platforms for biomimetic tissue engineering. Advanced Materials, 2015. 27(19): p. 2989-2995.

22. Yánez, A., et al., Compressive behaviour of gyroid lattice structures for human cancellous bone implant applications. Materials Science and Engineering C, 2016. 68: p. 445-448.

23. Wang, Q., et al., Antimicrobial Cu-bearing stainless steel scaffolds. Materials Science and Engineering C, 2016. 68: p. 519-522.

24. Luo, Y., et al., Synthesis and Biological Evaluation of Well-Defined Poly(propylene fumarate) Oligomers and Their Use in 3D Printed Scaffolds. Biomacromolecules, 2016. 17(2): p. 690-697.

25. Mauck, S.C., et al., Biorenewable Tough Blends of Polylactide and Acrylated Epoxidized Soybean Oil Compatibilized by a Polylactide Star Polymer. Macromolecules, 2016. 49(5): p. 1605-1615.

26. Schneider, C.A., W.S. Rasband, and K.W. Eliceiri, NIH Image to ImageJ: 25 years of image analysis. Nat Meth, 2012. 9(7): p. 671-675.

27. Guyot, Y., et al., A computational model for cell/ECM growth on 3D surfaces using the level set method: a bone tissue engineering case study. Biomechanics and Modeling in Mechanobiology, 2014. 13(6): p. 1361-1371.

28. Guyot, Y., et al., Coupling curvature-dependent and shear stress-stimulated neotissue growth in dynamic bioreactor cultures: a 3D computational model of a complete scaffold.

Biomechanics and Modeling in Mechanobiology, 2016. 15(1): p. 169-180.

29. Janson, I.A. and A.J. Putnam, Extracellular matrix elasticity and topography: Material-based cues that affect cell function via conserved mechanisms. Journal of Biomedical Materials Research Part A, 2015. 103(3): p. 1246-1258.

30. Dalby, M.J., et al., Nucleus alignment and cell signaling in fibroblasts: response to a microgrooved topography. Experimental Cell Research, 2003. 284(2): p. 272-280.

31. Charest, J.L., A.J. García, and W.P. King, Myoblast alignment and differentiation on cell culture substrates with microscale topography and model chemistries. Biomaterials, 2007. 28(13): p. 2202-2210.

32. Heinl, P., et al., Cellular Ti-6Al-4V structures with interconnected macro porosity for bone implants fabricated by selective electron beam melting. Acta Biomaterialia, 2008. 4(5): $p$. 1536-1544.

33. Guarino, V., M. Marrese, and L. Ambrosio, Chemical and Physical Properties of Polymers for Biomedical Use, in Advanced Polymers in Medicine, F. Puoci, Editor. 2015, Springer International Publishing: Cham. p. 67-90.

34. Wu, W., et al., Influence of Layer Thickness and Raster Angle on the Mechanical Properties of 3D-Printed PEEK and a Comparative Mechanical Study between PEEK and ABS. Materials, 2015. 8(9): p. 5271.

35. Pia, G., et al., Gyroidal structures as approximants to nanoporous metal foams: clues from mechanical properties. Journal of Materials Science, 2017. 52(2): p. 1106-1122.

36. Speirs, M., et al., Fatigue behaviour of NiTi shape memory alloy scaffolds produced by SLM, a unit cell design comparison. Journal of the Mechanical Behavior of Biomedical Materials, 2017. 70: p. 53-59.

37. Woodruff, M.A. and D.W. Hutmacher, The return of a forgotten polymer-Polycaprolactone in the 21st century. Progress in Polymer Science (Oxford), 2010. 35(10): p. 1217-1256.

38. Felfel, R.M., et al., Accelerated in vitro degradation properties of polylactic acid/phosphate glass fibre composites. Journal of Materials Science, 2015. 50(11): p. 3942-3955.

39. Höglund, A., K. Odelius, and A.-C. Albertsson, Crucial Differences in the Hydrolytic Degradation between Industrial Polylactide and Laboratory-Scale Poly(L-lactide). ACS Applied Materials \& Interfaces, 2012. 4(5): p. 2788-2793.

40. Ravi, P., P.S. Shiakolas, and T.R. Welch, Poly-l-lactic acid: Pellets to fiber to fused filament fabricated scaffolds, and scaffold weight loss study. Additive Manufacturing, 2017. 16: p. 167-176. 


\section{Internet reference}

1. http://www.3dvia.com/content/DCA841D2E4F6C8DA ( attached licence:

http://creativecommons.org/licenses/by-nc/2.5/ ) (last view July 2016) 